documented language emerged between notes of patients whose deaths were, and patients whose deaths were not supported by LCP-use, we carried out a secondary, content analysis of random subsets of each of these two groups of notes.

Aims To assess whether relatives were informed that the patient is dying and to compare differences in documentation language when the LCP was and was not used.

Results Of 96 adult deaths during October 2012, 83 case notes were available for analysis. After excluding 27 (33\%) sudden or unexpected deaths, the remaining 56 (67\%) expected deaths split into two sets: $41(73 \%)$ supported by LCP, and $15(27 \%)$ not supported. In both sets, the relatives had been informed that the patient was dying: LCP, 100\%; and non-LCP, 93\%. However, content analysis revealed that hedged language (terms such as 'unlikely to survive', 'looks to be dying', 'un-survivable') was more frequently documented in deaths unsupported by the LCP, compared to those supported by the LCP, where terms used in the medical notes connoted a greater degree of certainty, e.g. 'is dying', 'not responding to treatment.'

Conclusion BTHFT' staff document communication about dying well, despite contrary, adverse media reporting. Content analysis results suggest that hedged documentation language is associated with lower likelihood of integrated care pathway use for dying patients.

\section{P 158 PREDICTING WHEN THE LCP MIGHT NOT BE USED?: AN AUDIT OF COMMUNICATION WITH SECONDARY, CONTENT ANALYSIS OF LANGUAGE}

Milind Arolker, Margaret Ford, Susan Marshall. HOSPITAL PALLIATIVE CARE TEAM, BRADFORD TEACHING HOSPITALS NHS FOUNDATION TRUST, Bradford, UK

10.1136/bmjspcare-2014-000654.199

Background Bradford Teaching Hospitals NHS Foundation Trust (BTHFT) provides acute care services and comprises around 1200 beds. Although it participates in the National Care of the Dying Audit (NCDAH), this recurring process previously examined documentation just within the Liverpool Care Pathway (LCP) document. Following adverse media interest in the LCP, we carried out a retrospective casenote audit of deaths examining all documentation surrounding the death of the patients; data was collected from medical, nursing and, if used, LCP documentation regarding communication for patients and relatives about dying in general. When differences in the 